\title{
Effect of the Concentration of Dissolvent and Reductor Agent in the Synthesis of Tungsten-based Structures Using Microwave Approach and It Use as Catalysts in Dmf Hydrogen Donor
}

Miriam Estrada Flores ( $\sim$ mestradaf0400@ipn.mx)

Instituto Politécnico Nacional https://orcid.org/0000-0003-3005-2939

Tonantzi Guzmán-Barba

Instituto Politécnico Nacional

Carmen M. Reza-SanGermán

Instituto Politécnico Nacional

María Elena Manríquez-Ramírez

Instituto Politécnico Nacional

Oscar G. Rojas-Valencia

Instituto Politécnico Nacional

Cecilia Encarnación-Gómez

Universidad Juárez Autónoma de Tabasco

María Guadalupe Hernández-Cruz

Universidad Juárez Autónoma de Tabasco

Research Article

Keywords: Microwave approach, tungsten oxide, tungsten disulfide, hydrogen donor, DMF

Posted Date: March 1st, 2022

DOI: https://doi.org/10.21203/rs.3.rs-1397175/v1

License: (c) (1) This work is licensed under a Creative Commons Attribution 4.0 International License.

Read Full License 


\section{Abstract}

Tungsten-based structures by microwave approach were synthesized. Four different essays were used to analyze the effect of some synthesis variables; in two of them the effect of the concentration of the solvent was studied using pure ethylene glycol and a mix of ethylene glycol and water, while the other samples the effect of the presence of reductor agent using sodium borohydride was studied. All the samples were prepared using as precursor ammonium tetrathiotungstate and sulfur in excess to incentive the growth of structures enriched in this element, for each synthesis 1 minute of irradiation was used with microwave power level of 1200 watts and frequency of $2.64 \mathrm{GHz}$. The structural, morphological, and optical characterizations were carried out with Scanning Electron Microscopy (SEM), Transmission Electron Microscopy (TEM), infrared and uv-vis spectroscopies. By SEM, all the samples demonstrated differences in the size and morphology derivate of the modification of the experimental variables, the order of magnitude of the synthesized materials ranged from $0.01 \mu \mathrm{m}$ until $6.5 \mu \mathrm{m}$, with morphologies of tetragonal prisms, flakes, irregular agglomerates or flower-like depends on the characteristics of the precursor solution. TEM lets to determine the composition of each sample, corroborated also by IR and Diffuse Reflectance spectroscopies. The four samples were used as catalysts in the study of dimethylformamide (DMF) hydrogen donor.

\section{Full Text}

This preprint is available for download as a PDF.

\section{Tables}

Table 1

Loss Tangent (tan $\delta$ ) of different solvents at $2.45 \mathrm{GHz}$ and $20^{\circ} \mathrm{C}$ [20] 


\begin{tabular}{|llllll|}
\hline High & & Medium & & Low & \\
\hline Solvent & $\tan \delta$ & Solvent & $\tan \delta$ & Solvent & $\tan \delta$ \\
\hline Ethylene glycol & 1.17 & 2-Butanol & 0.45 & Chloroform & 0.091 \\
\hline Ethanol & 0.94 & 2-Methoxyethanol & 0.41 & Ethyl acetate & 0.059 \\
\hline 2-Propanol & 0.8 & 1-Hexanol & 0.34 & Acetone & 0.054 \\
\hline 1-Propanol & 0.76 & Dichlorobenzene & 0.28 & Dichloromethane & 0.042 \\
\hline Formic acid & 0.72 & Acetic acid & 0.17 & Toluene & 0.04 \\
\hline Benzyl alcohol & 0.67 & DMF & 0.16 & 2-Ethoxyethanol & 0.039 \\
\hline Methanol & 0.66 & Dichloroethane & 0.13 & Hexane & 0.02 \\
\hline Nitrobenzene & 0.59 & Water & 0.12 & o-Xylene & 0.018 \\
\hline 1-Butanol & 0.57 & Chlorobenzene & 0.1 & & \\
\hline Isobutanol & 0.52 & & & & \\
\hline
\end{tabular}

Table 2

Interplanar spacing (theorical and experimental) 


\begin{tabular}{|c|c|c|c|c|c|}
\hline Sample & $\begin{array}{l}\text { Measured } \\
\text { d } 0\end{array}$ & $\begin{array}{l}\text { PCPDF } \\
\text { Card }\end{array}$ & $\begin{array}{l}\text { Theorical d } \\
0\end{array}$ & hkl & $\begin{array}{l}\text { Crystalline } \\
\text { system }\end{array}$ \\
\hline \multirow{23}{*}{$\begin{array}{l}\text { Water - ethylene glycol with } \\
\mathrm{NaBH}_{4}\end{array}$} & 3.918 & \multirow[t]{23}{*}{$89-1287$} & 3.88 & 001 & \multirow[t]{23}{*}{ Tetragonal } \\
\hline & 3.684 & & 3.695 & 200 & \\
\hline & 3.436 & & 3.4353 & 101 & \\
\hline & 3.317 & & 3.3049 & 210 & \\
\hline & 3.116 & & 3.1151 & 111 & \\
\hline & 2.701 & & 2.6757 & 201 & \\
\hline & 2.638 & & 2.6127 & 220 & \\
\hline & 2.505 & & 2.5159 & 211 & \\
\hline & 2.341 & & 2.3369 & 310 & \\
\hline & 2.168 & & 2.1672 & 221 & \\
\hline & 2.089 & & 2.0796 & 301 & \\
\hline & 2.000 & & 2.0018 & 311 & \\
\hline & 1.798 & & 1.7923 & 410 & \\
\hline & 1.724 & & 1.7178 & 202 & \\
\hline & 1.665 & & 1.6680 & 401 & \\
\hline & 1.650 & & 1.6524 & 420 & \\
\hline & 1.629 & & 1.6271 & 411 & \\
\hline & 1.557 & & 1.5575 & 222 & \\
\hline & 1.517 & & 1.5203 & 421 & \\
\hline & 1.439 & & 1.4493 & 510 & \\
\hline & 1.243 & & 1.2380 & 441 & \\
\hline & 1.119 & & 1.1188 & 521 & \\
\hline & 1.079 & & 1.0937 & 323 & \\
\hline \multirow{5}{*}{$\begin{array}{l}\text { Water - ethylene glycol without } \\
\mathrm{NaBH}_{4}\end{array}$} & 3.842 & \multirow[t]{5}{*}{$89-1287$} & 3.88 & 001 & \multirow[t]{5}{*}{ Tetragonal } \\
\hline & 3.711 & & 3.695 & 200 & \\
\hline & 3.484 & & 3.4353 & 101 & \\
\hline & 3.297 & & 3.3049 & 210 & \\
\hline & 3.126 & & 3.1151 & 111 & \\
\hline
\end{tabular}




\begin{tabular}{|c|c|c|c|c|c|}
\hline & & & & \\
\hline & 2.703 & & 2.6757 & 201 & \\
\hline & 2.602 & & 2.6127 & 220 & \\
\hline & 2.475 & & 2.5159 & 211 & \\
\hline & 2.355 & & 2.3369 & 310 & \\
\hline & 2.177 & & 2.1672 & 221 & \\
\hline & 1.982 & & 2.0018 & 311 & \\
\hline & 1.839 & & 1.8475 & 400 & \\
\hline & 1.810 & & 1.8187 & 112 & \\
\hline & 1.657 & & 1.6524 & 420 & \\
\hline & 1.583 & & 1.5890 & 331 & \\
\hline & 1.5246 & & 1.5203 & 421 & \\
\hline & 1.477 & & 1.480 & 430 & \\
\hline & 1.439 & & 1.4493 & 510 & \\
\hline \multirow[t]{12}{*}{ Ethylene glycol without $\mathrm{NaBH}_{4}$} & 3.875 & \multirow[t]{12}{*}{$20-1324$} & 3.85 & 001 & \multirow[t]{12}{*}{ Orthorhombic } \\
\hline & 3.728 & & 3.75 & 020 & \\
\hline & 3.597 & & 3.69 & 200 & \\
\hline & 3.468 & & 3.43 & 011 & \\
\hline & 3.338 & & 3.35 & 120 & \\
\hline & 2.700 & & 2.686 & 021 & \\
\hline & 2.635 & & 2.633 & 220 & \\
\hline & 2.161 & & 2.173 & 221 & \\
\hline & 1.900 & & 1.923 & 002 & \\
\hline & 1.836 & & 1.846 & 400 & \\
\hline & 1.798 & & 1.806 & 112 & \\
\hline & 1.65 & & 1.657 & 420 & \\
\hline \multirow[t]{3}{*}{ Ethylene glycol with $\mathrm{NaBH}_{4}$} & 6.165 & \multirow[t]{3}{*}{$84-1398$} & 6.1615 & 002 & \multirow[t]{3}{*}{ Hexagonal } \\
\hline & 3.093 & & 3.0807 & 004 & \\
\hline & 2.641 & & 2.6660 & 101 & \\
\hline
\end{tabular}


Table 3

Values of band gap reported of WO3, WO2 and WS2

\begin{tabular}{|c|c|c|}
\hline Rename & Crystalline structure & Reported Band Gap (eV) \\
\hline$a-\mathrm{WO}_{3}$ & Tetragonal & $2.9-3.21 \mathrm{eV}[38-40]$ \\
\hline $\mathrm{B}-\mathrm{WO}_{3}$ & Orthorhombic & $2.52 \mathrm{eV}[41]$ \\
\hline$y-\mathrm{WO}_{3}$ & Monoclinic & $1.98 \mathrm{eV}$ y $2.58 \mathrm{eV}, 2.4 \mathrm{eV}$ [39] \\
\hline$\delta-\mathrm{WO}_{3}$ & Triclinic & $2.43 \mathrm{eV}$ y $2.60 \mathrm{eV}$ [37] \\
\hline $\mathbb{Q}-\mathrm{WO}_{3}$ & Monoclinic & $2.55 \mathrm{eV}-2.85 \mathrm{eV}$ [37] \\
\hline $\mathrm{WO}_{2}$ & Hexagonal & $1.863 \mathrm{eV}[42]$ \\
\hline \multirow[t]{2}{*}{$\mathrm{WS}_{2}$} & Hexagonal & 1.3 - $1.94 \mathrm{eV}$ [43], [44] \\
\hline & & $1.51-2.03 \mathrm{eV}[45]$ \\
\hline
\end{tabular}

\section{Figures}

\section{Figure 1}

SEM micrographs of the samples: a) water-ethylene glycol without $\mathrm{NaBH} 4$, b) ethylene glycol without $\mathrm{NaBH} 4, \mathrm{c})$ water-ethylene glycol with $\mathrm{NaBH} 4$ and d) ethylene glycol with $\mathrm{NaBH} 4$

\section{Figure 2}

TEM micrographs of the samples: a) water-ethylene glycol without $\mathrm{NaBH} 4$, b) water-ethylene glycol with $\mathrm{NaBH} 4, \mathrm{c}$ ) ethylene glycol without $\mathrm{NaBH} 4$ and d) ethylene glycol with $\mathrm{NaBH} 4$

\section{Figure 3}

General IR spectrum of the samples, with functional groups identification. 
IR spectral of the different samples: a) ethylene glycol - water without $\mathrm{NaBH} 4, \mathrm{~b}$ ) ethylene glycol - water with $\mathrm{NaBH} 4$, c) ethylene glycol without $\mathrm{NaBH} 4$, d) ethylene glycol with $\mathrm{NaBH} 4$. Before irradiation - black lines and after irradiation - red lines.

\section{Figure 5}

Band Gap of the simples using the Kubelka Munk function: a) ethylene glycol - water without $\mathrm{NaBH} 4, \mathrm{~b}$ ) ethylene glycol - water with $\mathrm{NaBH} 4$, c) ethylene glycol without $\mathrm{NaBH} 4$, d) ethylene glycol with $\mathrm{NaBH} 4$

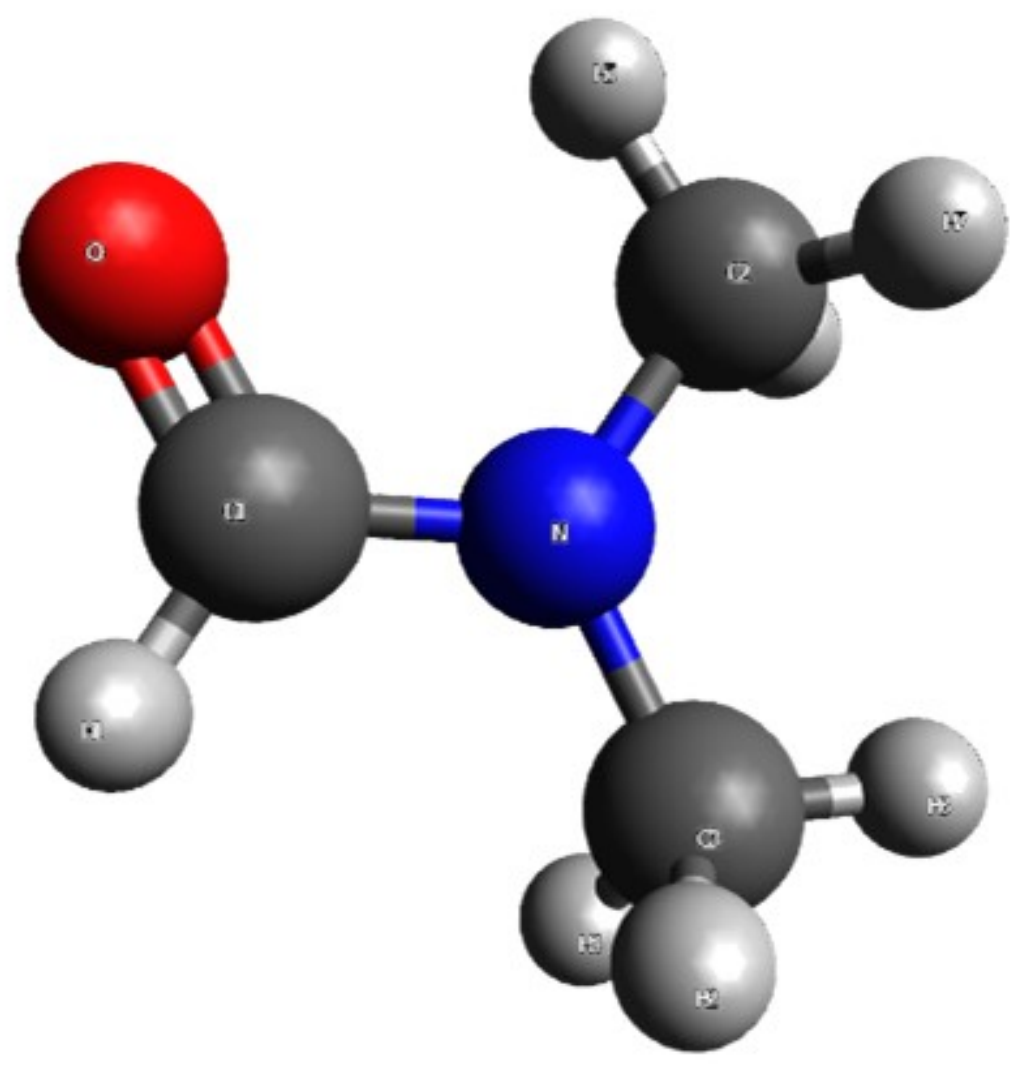

Figure 6

DMF structure

Figure 7

IR spectrums of DMF 